\title{
The molecular analysis of synonymy among medically important yeasts within the genus Candida
}

\author{
B. L. WiCKes, ${ }^{1}$ J. B. Hicks, ${ }^{2}$ W. G. Merz ${ }^{3}$ and K. J. KwON-ChUNG ${ }^{1 *}$ \\ ${ }^{1}$ Laboratory of Clinical Investigation, National Institute of Allergy and Infectious Diseases, Bethesda, MD 20892, USA \\ ${ }^{2}$ Icos Inc., 22021 20th Ave SE, Bothell, WA 98021, USA \\ ${ }^{3}$ Department of Laboratory Medicine, The Johns Hopkins Medical Institute, Meyer B1-193, Baltimore, MD 21205, USA
}

(Received 18 November 1991; accepted 21 January 1992)

\begin{abstract}
Three sets of medically important yeasts, Candida albicans, $C$. tropicalis, and $C$. krusei, were compared with their putative synonyms (C. langeronii and C. claussenii, $C$. paratropicalis, and Itssatchenkia orientalis, respectively) to determine if these synonyms are genetically distinguishable from each other. Pulsed-field electrophoresis and hybridization to species-specific probes were used to accomplish this goal. The species-specific probes for C. albicans and $C$. tropicalis have been previously described (27A and CT13.8, respectively) whereas the probe for C. krusei (CK3) was cloned in this study. No distinguishing characteristics between synonyms were identified, thus supporting the current taxonomic treatment of these organisms.
\end{abstract}

\section{Introduction}

Classification of the yeasts relies upon testing a selected panel of biochemical reactions, with a determination made regarding the identity of the isolate supported, in part, by morphological characteristics. Within any set of biochemical reactions for a given species there are bound to be a few which can be variable, depending on the isolate. Problems arise when a reaction viewed as 'typical' (all isolates of that species respond the same) is atypical, or when the number of variable reactions (' + or - ') for a given species becomes large.

The genus Candida has been described as a 'taxonomic pit' (Odds, 1987), a reflection of the diverse nature of the organisms placed there. This genus has been frequently reclassified with respect to the composition of its members. Species have been added, subtracted, and renamed. Much of this activity has involved members that are closely related, but are variable in too many characteristics to classify them as biochemically homogeneous. Compounding the difficulty of proper classification is the potential for variation, of particular significance for the medically important members of this genus. This variability can be manifested in genetic, biochemical or morphological properties.

- Author for correspondence. Tel. (301) 496 1602; fax. (301) 480 0050 .

Abbreviation: CHEF, contour-clamped homogeneous electric field.
A partial solution to this problem has been the employment of DNA-DNA homology for the determination of relatedness by assaying an unknown isolate against a 'typical' (usually the type culture) control (Meyer, 1979). This technique has proven an invaluable tool to fungal taxonomists and has by and large confirmed, the excellent job that classical mycologists have done with regard to taxonomy. While this technique is arguably the single most important standard with regard to an accurate determination of relatedness, there are instances in which proper taxonomic conclusions may require additional techniques. For example, DNA-DNA homology, unless performed with unique sequences, results in chimpanzees and humans having a homology of 95\% (Kohne et al., 1972). On the other hand, if there are numerous inversions present within the genome, this could give an erroneously low value. Also, DNA-DNA homology requires the use of special skills and inclusion of the type culture in each assay. Finally, DNA-DNA homology generally does not detect the presence of subgroups. While this is not important for simple variants, the presence of a distinct subgroup or cluster should be determined in order to account for any potentially important distinguishing characteristics, especially if they are of diagnostic value or play a role in pathogenicity.

We have utilized two molecular techniques, hybridization to species-specific probes and pulsed-field gel electrophoresis, to study the relationship among organ- 
isms in three different groups of medically important yeasts. In this study, we compared Candida tropicalis with $C$. paratropicalis, C. krusei with Itssatchenkia orientalis, and $C$. albicans with $C$. langeronii and $C$. claussenii using these techniques. C. paratropicalis possesses $>95 \%$ DNA-DNA homology with $C$. tropicalis, but can be distinguished by its inability to ferment sucrose and melezitose, and assimilation of L-arabinose (Baker et al., 1981). C. krusei is considered to be the haploid state of $I$. orientalis by DNA-DNA homology (Kurtzman et al., 1980), yet in our experience no clinical isolates of $C$. krusei ever produced an ascomycetous state or mated with tester strains of $I$. orientalis. C. claussenii and $C$. langeronii are treated as synonyms of $C$. albicans. C. claussenii is considered to be a germ-tube-negative mutant (Lodder \& Kreger-van Rij, 1952), while $C$. langeronii is considered to be a chlamydosporenegative mutant (Uden \& Buckley, 1970).

These yeasts were chosen for study because recent molecular and genetic analyses of the relationship of C. albicans to C. stellatoidea in this laboratory (KwonChung et al., 1988, 1989), and others (Kamiyama et al., 1989), have shown that Type I C. stellatoidea comprises a distinct subgroup of $C$. albicans although this was not recognized by DNA-DNA homology. In this study we have cloned a species-specific sequence for the detection of $C$. krusei, and investigated the possibility that within the synonymy of $C$. tropicalis, $C$. albicans, and C. krusei, there are distinct subgroups which should be recognized as being more than atypical variants.

\section{Methods}

Strains and probes. All strains and probes used in this study are listed in Table 1. All isolates were tested for identity using the API 20C system (Analytab Inc.) in addition to other diagnostic criteria (morphology, germ tube production). The species-specific probes CT13.8 for C. tropicalis, 27A for C. albicans and CK13 for C. krusei were used to determine strain relatedness, and where possible, DNA fingerprints. 27A was cloned by S. Scherer and D. A. Stevens and has been described extensively (Fox et al., 1989; Scherer \& Stevens, 1988). CK 13 was cloned using the folowing protocol. Genomic DNA was partially digested with Sau3A and ligated into Bluescribe + (Stratagene) opened at the BamHI site. After transformation into Escherichia coli, individual colonies were picked to a grid on nitrocellulose filters. After growth on the filters, the colonies were lysed in situ and hybridized in $4 \times$ SSC $(0.6 \mathrm{M}$-sodium chloride, $0.06 \mathrm{M}$-sodium citrate, pH 7.0) containing $10 \mu \mathrm{g}$ salmon sperm DNA ml ${ }^{-1}$ and $0.2 \%$ lauryl sarkosinate. Unfractionated DNA used to make the library was ${ }^{32} \mathbf{P}$ labelled by random priming (Amersham) and used to probe the filters. Colonies showing hybridization signals greater than background levels were rescreened with a clone containing $C$. albicans ribosomal DNA. DNA was prepared from the colonies that did not hybridize with this clone and used to probe Southern filters containing DNA digests of numerous Candida species. The clone hybridizing most intensely, and to the largest number of bands in C. krusei, but not to the others, was selected for further study. CT13.8 was previously cloned using the
Table 1. Cultures and probes used in this study

\begin{tabular}{lcl}
\hline \hline Organism & Culture No. & \multicolumn{1}{c}{ Source*/no. } \\
\hline C. albicans & B-311 & NIH \\
C. albicans & B-4207 & NIH \\
C. albicans (type) & B-4497 & ATCC 18804 \\
C. stellatoidea (type) & B-4252 & ATCC 11006 \\
C. tropicalis (type) & B-4295 & ATCC 750 \\
C. tropicalis & B-4449 & D. Ahearn, CDC-59 \\
C. tropicalis & B-4414 & ATCC 38292 \\
C. tropicalis & B-4415 & ATCC 14246 \\
C. paratropicalis (type) & B-4434 & ATCC 42678 \\
C. paratropicalis & B-4436 & D. Pincus, 81MR 457 \\
C. krusei (type) & B-4494 & ATCC 6258 \\
C. krusei & 3168 & NIH \\
I. orientalis (type) & B-4496 & C. Kurtzman, Y-8281 \\
C. kefyr (type) & B-4298 & ATCC 42265 \\
C. pseudotropicalis (type) & B-4296 & ATCC 4135 \\
C. parapsilosis (type) & B-4299 & ATCC 22019 \\
C. parapsilosis & 3175 & NIH \\
S. cerevisiae & B-4262 & R. Wickner, S288C \\
C. claussenii & B-4491 & ATCC 18814 \\
C. langeronii & B-4492 & ATCC 22972 \\
\hline \hline Sequence & & \\
\hline 27A & Species & Source* \\
CT13.8 & C. albicans & S. Scherer \\
CK13 & C. tropicalis & J. Hicks \\
\hline \hline
\end{tabular}

* NIH, National Institutes of Health, Bethesda, MD, USA; ATCC, American Type Culture Collection, Rockville, MD, USA; D. Ahearn, Dept of Biology, Georgia State University, Atlanta, GA, USA; D. Pincus, Analytab Products, Plainview, NY, USA; C. Kurtzman, Agricultural Research Culture Collection, USDA, Peoria, IL, USA; R. Wickner, NIDDK, Bethesda, MD, USA; S. Scherer, Dept of Microbiology, University of Minnesota Medical School, Minneapolis, MN, USA; J. Hicks, Icos, Inc., Bothell, WA, USA.

same strategy, and has been successfully used as a species-specific probe (Soll et al., 1988).

Pulsed-field electrophoresis. Agarose plugs were prepared using the method described by Wickes et al. (1991a). Electrophoresis conditions were as follows. For all isolates, plugs were melted at $67^{\circ} \mathrm{C}$ and loaded into a $\mathbf{0 . 4} \%$ chromosomal-grade agarose (CGA) gel (Bio-Rad) made up in $0.5 \times$ TBE (TBE is $89 \mathrm{~mm}$-Tris/borate, $2 \mathrm{mM}$-EDTA, pH 8). For C. tropicalis and C. paratropicalis, dual ramped switch times were used for separation, beginning with $180-300 \mathrm{~s}$ for $15 \mathrm{~h}$, and followed by 500 $1000 \mathrm{~s}$ for $99 \mathrm{~h}$ at $60 \mathrm{~V}$. C. krusei and I. orientalis chromosomes were separated using a $0.5 \%$ CGA gel with dual ramped switch times of 180 $300 \mathrm{~s}$ for $25 \mathrm{~h}$ followed by $420-900 \mathrm{~s}$ for $95 \mathrm{~h}$ at $65 \mathrm{~V}$. C. claussenii, C. langeronii and C. albicans chromosomes were separated in a $0.5 \%$ CGA gel using dual ramped switch times of $180-300 \mathrm{~s}$ for $25 \mathrm{~h}$ followed by $420-900 \mathrm{~s}$ for $95 \mathrm{~h}$ at $65 \mathrm{~V}$. All runs were performed using a Bio-Rad CHEF DRII apparatus in $0.5 \times$ TBE running buffer kept at a constant temperature of $12^{\circ} \mathrm{C}$.

DNA extraction and restriction analysis. DNA was extracted from al isolates using a modification of a method previously described for Cryptococcus neoformans (Varma \& Kwon-Chung, 1991). In this method, cells were harvested after $20 \mathrm{~h}$ of growth on Sabouraud's dextrose agar plates. Zymolyase 20T (ICN Immunobiologicals) was substituted as the spheroplasting enzyme by adding $400 \mu \mathrm{l}$ of a $45 \mathrm{mg}$ $\mathrm{ml}^{-1}$ stock solution to the cell suspensions. 
Restriction digests were performed according to the manufacturer's specifications. For each sample, $1 \mu \mathrm{g}$ DNA was digested with $5 \mathrm{U}$ of the appropriate enzyme (Gibco-BRL) for $4 \mathrm{~h}$ at $37^{\circ} \mathrm{C}$. Samples were electrophoresed in a $0.8 \%$ agarose gel at $1.5 \mathrm{~V} \mathrm{~cm}^{-1}$ for $20 \mathrm{~h}$. Blotting and Southern hybridizations were performed according to standard methods (Maniatis et al., 1982).

\section{Results}

\section{Hybridization to species-specific probes}

C. tropicalis vs $C$. paratropicalis. The probe CT13.8 is a $2.4 \mathrm{~kb}$, moderately repetitive, non-rDNA sequence useful for distinguishing isolates of $C$. tropicalis from other species of Candida, and each other. It has previously been used to distinguish clinical isolates of $C$. tropicalis from C. albicans (Soll et al., 1988). When the probe was hybridized to Southern blots of digested genomic DNA from numerous yeast isolates, extensive hybridization was observed only for $C$. tropicalis and $C$. paratropicalis (Fig. 1). Both of these isolates yielded 15-20 bands ranging in size from approximately $13 \mathrm{~kb}$ to $1.75 \mathrm{~kb}$. The other isolates either did not hybridize, or yielded only a limited number of bands. This probe was able to distinguish $C$. tropicalis from other isolates of Candida, including $C$. albicans. However, it was not able to distinguish $C$. tropicalis from $C$. paratropicalis, by either band number or intensity.

C. krusei vs I. orientalis. The probe CK13 is a $0.9 \mathrm{~kb}$ fragment that was isolated from C. krusei and was found to be useful as a species-specific probe, although not discriminatory enough for intraspecies fingerprinting. This is probably due to the small size of the fragment. Hybridization of this probe to Southern blots of digested genomic DNA from multiple Candida isolates resulted in strong hybridization to $C$. krusei and $I$. orientalis DNA in the 8-10 kb size range, but not to other Candida species (Fig. 2). Ocassionally, weak hybridization signals to one or more non-krusei bands were observed, but not enough to interfere with detection of $C$. krusei isolates. As in the case of $C$. tropicalis and $C$. paratropicalis, $I$. orientalis could not be distinguished from $C$. krusei using this procedure.

C. albicans vs $C$. langeronii and $C$. claussenii. In order to compare these three isolates, the probe $27 \mathrm{~A}$ was used. This probe has been shown to be a moderately repetitive, non-rDNA, species-specific sequence which hybridizes only to C. albicans DNA. Because of its repetitive nature, it is also useful for intraspecies discrimination since only the most closely related (clonal) strains display the same restriction pattern. It is therefore a potentially useful epidemiological tool. When this probe was hybridized to genomic digests of $C$. albicans, C. langeronii and

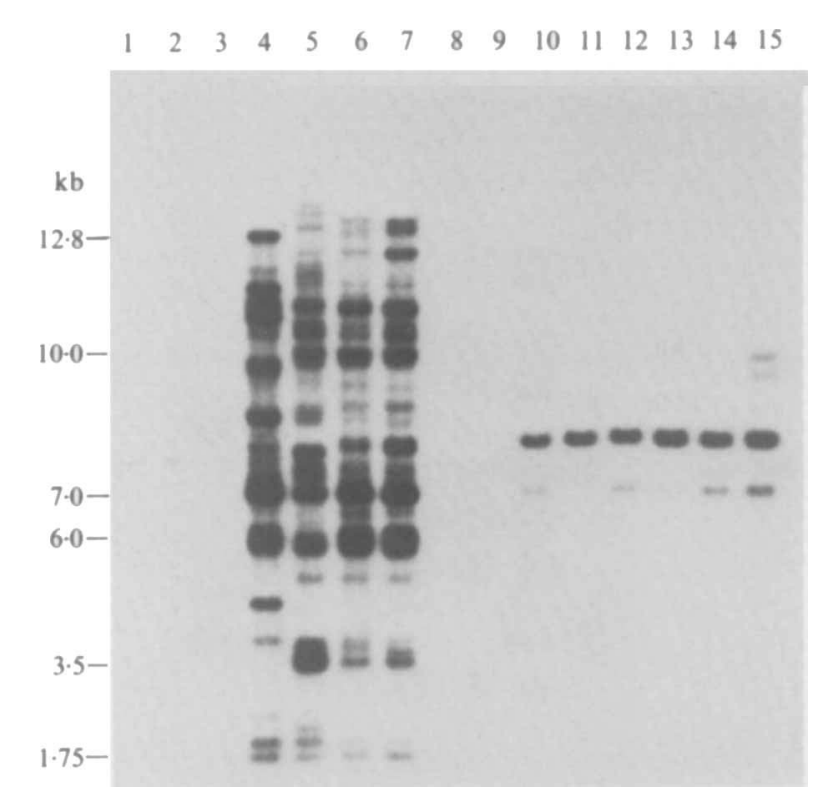

Fig. 1. Hybridization of EcoRI-digested DNA to CT13.8, a C. tropicalis, species-specific probe. Lanes: 1, C. albicans B-311 serotype A; 2, C. albicans B-4207 serotype B; 3, C. stellatoidea B-4252; 4, C. tropicalis B-4295; 5, C. tropicalis B-4449; 6, C. paratropicalis B-4434; 7, C. paratropicalis B-4436; 8, C. krusei B-4494; 9, C. krusei $3168 ; 10$, I. orientalis B-4496; 11, C. kefyr B-4298; 12, C. pseudotropicalis B-4296; 13, C. parapsilosis B-4299; 14, C. parapsilosis $3175 ; 15$, Saccharomyces cerevisiae B-4262.

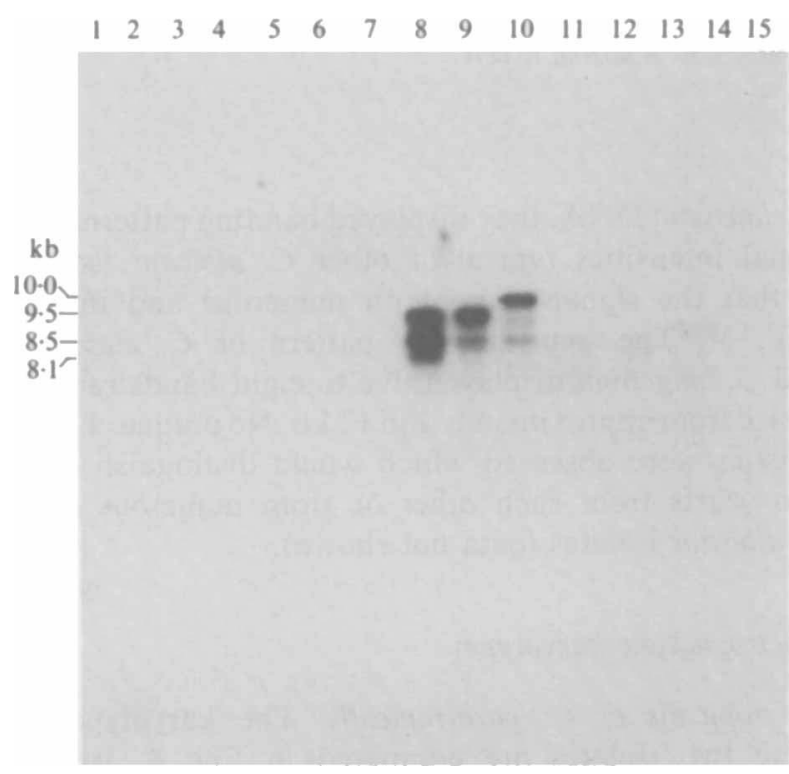

Fig. 2. Hybridization of EcoRI-digested DNA to CK13, a C. krusei, species-specific probe. Lanes: 1,C. albicans B-311 serotype A; 2, C. albicans B-4207 serotype B; 3, C. stellatoidea B-4252; 4, C. tropicalis B-4295; 5, C. tropicalis B-4449; 6, C. paratropicalis B-4434; 7 , C. paratropicalis B-4436; 8, C. krusei B-4494; 9, C. krusei 3168; 10 , I. orientalis B-4496; 11, C. kefyr B-4298; 12, C. pseudotropicalis B-4296; 13, C. parapsilosis B-4299; 14, C. parapsilosis $3175 ; 15$, S. cerevisiae B-4262. 


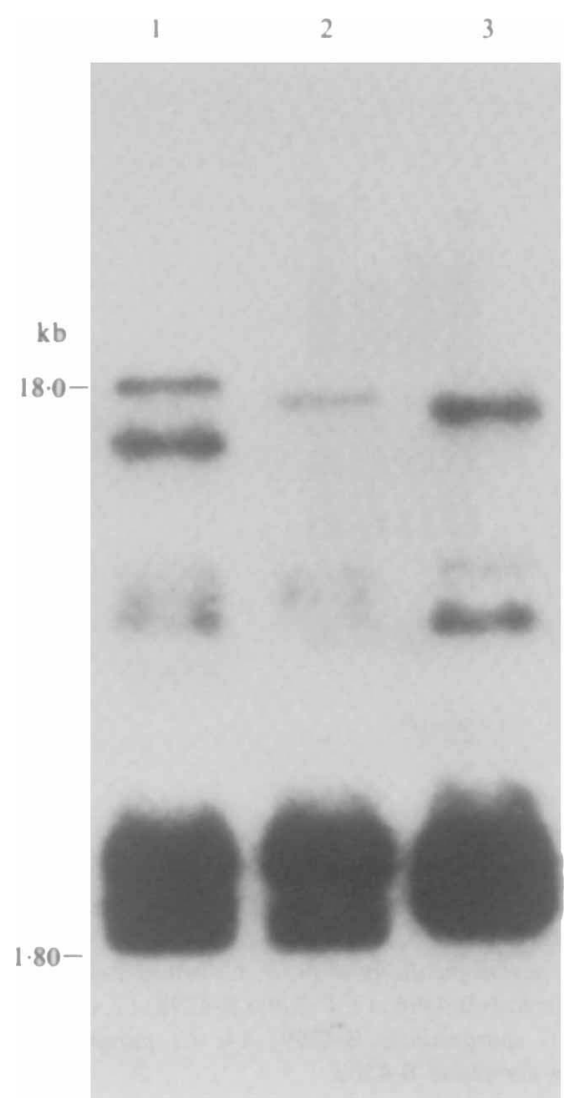

Fig. 3. Hybridization of EcoRI-digested DNA to 27A, a C. albicans, species-specific probe. Lanes: 1, C. albicans B-4497; 2, C. langeronii B-4492; 3, C. claussenii B-4491.

C. claussenii DNA, they displayed banding patterns and signal intensities typical of other $C$. albicans isolates in that the signals were both numerous and intense (Fig. 3). The hybridization pattern of $C$. claussenii and $C$. langeronii displayed five to eight bands ranging in size from approximately 2 to $12 \mathrm{~kb}$. No unique characteristics were observed which would distinguish these two yeasts from each other or from numerous other C. albicans isolates (data not shown).

\section{Electrophoretic karyotypes}

C. tropicalis vs $C$. paratropicalis. The karyotypes of these two isolates are compared in Fig. 4. Isolates of $C$. paratropicalis had karyotype patterns indistinguishable from those of $C$. tropicalis. Additionally, both had the same pattern of chromosome sizes: two were approximately $1-1.5 \mathrm{Mb}$ in size and five were approximately $2.5-4 \mathrm{Mb}$ in size. No sexual cycle has been described in C. tropicalis, and it has been recently shown to be diploid (Picataggio et al., 1991), so each band

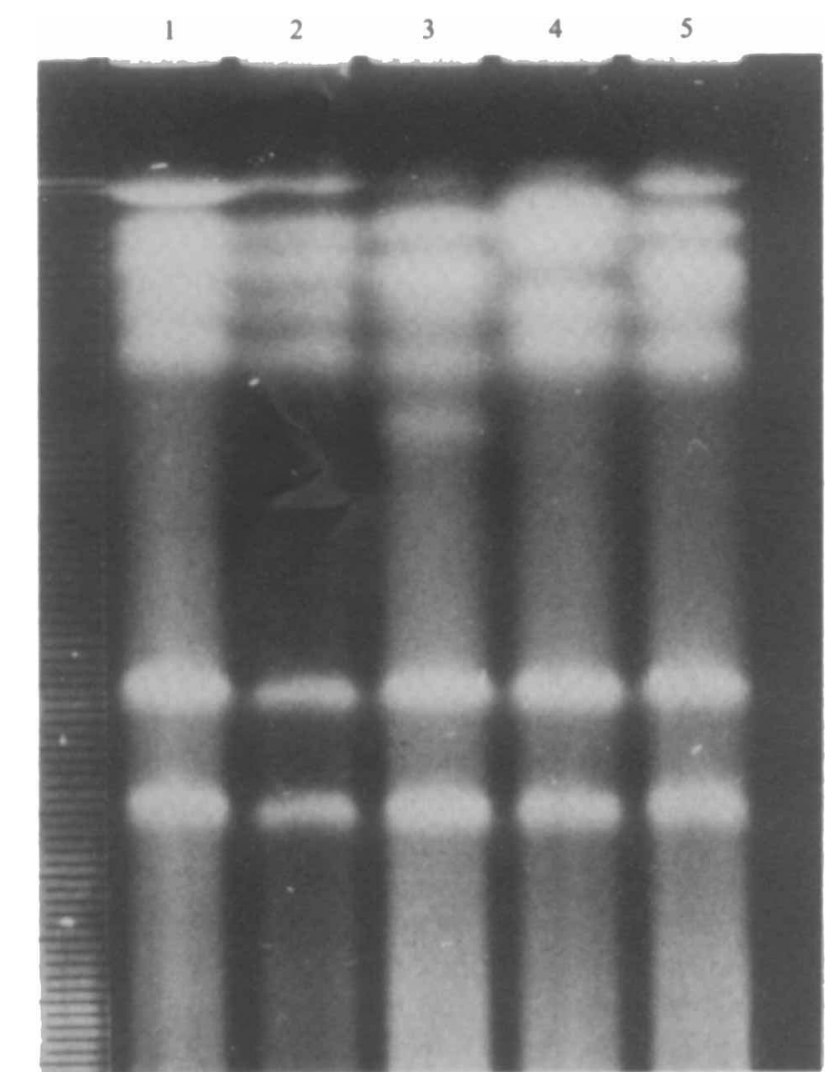

Fig. 4. CHEF electrophoresis of C. tropicalis and C. paratropicalis isolates. Lanes: 1, C. tropicalis B-4295; 2, C. tropicalis B-4414; 3, C. tropicalis B-4415; 4, C. paratropicalis B-4434; 5, C. paratropicalis B-4436.

probably represents two chromosomes. For both isolates, seven bands were observed and chromosomal polymorphisms were evident. As in $C$. albicans, the polymorphisms are probably due to separation of homologues and the unequal migration of the rDNA-containing chromosomes (Wickes et al., 1991b).

$C$. albicans os $C$. langeronii and $C$. claussenii. The karyotypes of these three isolates are shown in Fig. 5. These isolates possess chromosomes of a wide size range, with the karyotypes of $C$. langeronii and $C$. claussenii bearing a close resemblance to those of $C$. albicans. While the karyotype of $C$. albicans is known to be very heterogeneous, a general pattern exists within which the karyotypes of $C$. claussenii and $C$. langeronii fit. The chromosomes can generally be placed into three different groups. Chromosomes, 1, 2 and $\mathrm{R}$ (the rDNA-containing chromosome) comprise the largest and most variable class; chromosomes 3 and 4 are mid-sized and very typical of $C$. albicans, and chromosomes 5, 6 and 7 comprise the smallest class. While this general pattern is typical of C. albicans, there are exceptions. Deviation is 


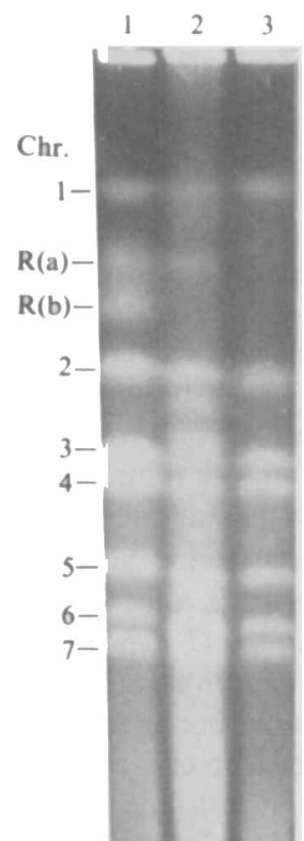

Fig. 5. CHEF electrophoresis of $C$. albicans, $C$. langeronii and $C$. claussenii isolates. Lanes: 1, C. albicans B-4497; 2, C. langeronii B-4492; 3, C. claussenii B-4491.

generally due to separation of homologues and/or differences in migration of the rDNA-containing chromosome. C. claussenii appears to lack one of the larger chromosomes, a phenomenon which we have also occasionally observed in some $C$. albicans isolates. This can result from extensive shearing of the $\mathrm{R}$ chromosome, possibly exacerbated by differences in size of this chromosome in a given population. This size difference is always manifested by fuzziness of the $\mathrm{R}$ chromosome, even in unsheared samples. The karyotype of $C$. langeronii possesses extra bands in the region of chromosomes 6,4 and 3 of $C$. albicans. No linkage mapping has been performed with these isolates so it cannot be determined if these extra bands are homologues. However, the general pattern resembles C. albicans, and the largest and smallest bands are consistent in size with their C. albicans counterpart.

C. krusei vs $I$. orientalis. The karyotypes of these two yeasts are shown in Fig. 6. They are nearly identical. For each isolate, we estimate the number of chromosomes to be five, based on the separation of four bands and the high intensity of the largest band, which suggests the presence of at least two chromosomes in that region. In contrast to $C$. albicans, there do not appear to be any midsized chromosomes. While the ploidy of this organism has not been confirmed, separation of homologous chromosomes was not observed.

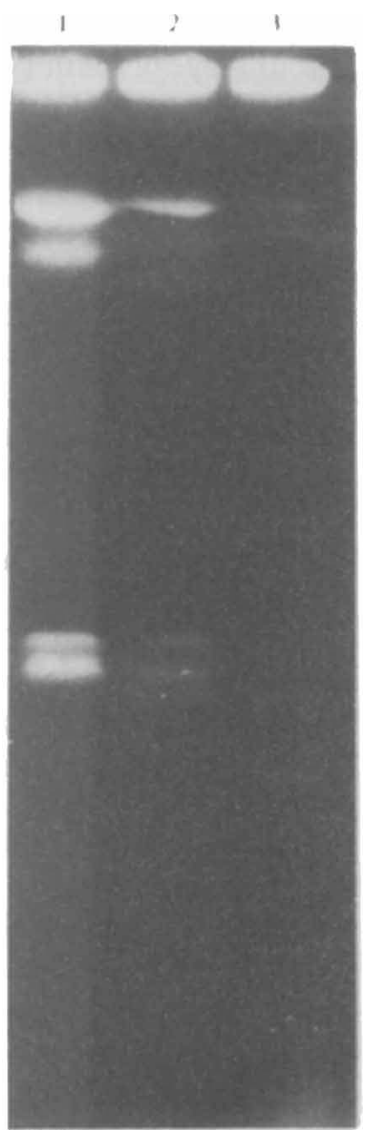

Fig. 6. CHEF electrophoresis of $C$. krusei and I. orientalis isolates. Lanes: 1, C. krusei B-4494; 2, I. orientalis B-4496; 3, C. albicans B-4497.

\section{Discussion}

The development of species-specific probes has enabled researchers to identify particular isolates of interest quickly and conclusively. A side benefit of these probes is that they are frequently useful for 'fingerprinting', which can establish clonal relationships; this makes them valuable epidemiological tools. For each species we investigated, we found that the species-specific probe for that species, hybridized to the putative synonymous isolate as well as to the control culture. Additionally, when useful fingerprint patterns were generated (27A, CT13.8), we found no distinct characteristics which could be used to distinguish the synonym from the type culture.

When we compared electrophoretic karyotypes within these groups, we also found that the putative synonyms did not possess karyotypes distinctly different from the species as a whole. C. krusei and I. orientalis were found to be identical; both possessed at least five chromosomes, which is in contrast to earlier reports of three 
(Iwaguchi et al., 1990; Suzuki et al., 1988). Based upon this observation, the absence of mating between clinical isolates of $C$. krusei and tester strains of I. orientalis at least, is not due to incompatible karyotypes if one considers I. orientalis to be heterothallic. Whether this organism is homothallic or heterothallic has not been clearly established (Kurtzman et al., 1980). The isolates of $C$. langeronii and $C$. claussenii could not be distinguished from $C$. albicans, although heterogeneity was observed within this group. It is rate to find two independent isolates which possess identical karyotypes, but it should be noted that only one isolate of each type was investigated. We do not know if all isolates of each type are identical or heterogeneous; however, all the chromosomes of $C$. claussenii and $C$. langeronii fell within the size ranges of the chromosomes of $C$. albicans. $C$. paratropicalis and $C$. tropicalis also had indistinguishable karyotypes which were comprised of seven bands. Previous reports have estimated the number of chromosomes of C. tropicalis to be eight (Fukuda et al., 1991; Suzuki et al., 1991) and six (Suzuki et al., 1988), respectively. It should be noted that in those papers, a single clinical isolate that was not the type culture was used, and extensive linkage mapping to determine if any of the bands could in fact be homologues which have separated was not done. This phenomenon is a frequent occurrence in C. albicans and is complicated by the observation of the $R$ chromosome being extremely variable in size. Together, these problems can complicate chromosome numbering, identification and quantification. In the light of the close taxonomic relationship of C. tropicalis to C. albicans, a final determination of chromosome number should include linkage mapping, since this will reveal any homologues which have separated, and also will account for both homologues.

The results of this study support the conclusions previously arrived at by classical yeast taxonomists. However, it should be emphasized that there may be other, more detailed parameters which would be more suitable for determining the proper relationship of these organisms with respect to others grouped under the same species. For taxonomists, this is obviously burdensome since it is only important to know 'who' an organism is in relation to others within the same genus. This is best done with as few characteristics as possible, in the interest of efficiency. The clinical mycologist, on the other hand, wants to know 'what' an organism is, and is therefore interested in any unusual characteristics it may possess since these may have an impact on treatment. Recent studies with Type I C. stellatoidea, for example, have shown that although it does not merit species status, this organism is not a simple variant of $C$. albicans in spite of $>95 \%$ DNA-DNA homology. In these studies, pulsed-field electrophoresis and DNA fingerprint pat- terns were the most significant indicators of Type I C. stellatoidea being a true subgroup of $C$. albicans. Additional evidence in support of this includes morphological (Martin et al., 1937), biochemical (Kamiyama et al., 1989), and genetic (Kwon-Chung et al., 1989) properties. Type II C. stellatoidea, on the other hand, has been shown to be a simple $\alpha$-glucosidase mutant of C. albicans (Kwon-Chung et al., 1990), and is indistinguishable in all other characteristics.

This study was based on the assumption that previously independent species reclassified as synonyms by DNA-DNA homology may actually qualify as a subgroup when tested using other molecular techniques. We investigated conspecific yeasts within the species C. albicans, C. tropicalis and C. krusei and found no evidence supportive of $C$. langeronii and $C$. claussenii (C. albicans), $C$. paratropicalis $(C$. tropicalis) being classified as anything but synonyms. In spite of the failure in mating between clinical strains of $C$. krusei and the tester strains of $I$. orientalis, our study showed that these were one and the same.

Future studies should investigate what parameters would be most effective or desirable for recognizing the existence of subgroups after biochemical similarity and DNA homology have been determined. Additionally, there may be other groups within the medically important yeasts that are worth investigating for subgroups, such as $C$. parapsilosis.

\section{References}

Baker, J. G., Salkin, I. F., Pincus, D. H. \& D'Amato, R. F. (1981). Candida paratropicalis, a new species of Candida. Mycotaxon 13, 115-119.

FoX, B. C., Mobley, H. L. T. \& W ADE, J. C. (1989). The use of a DNA probe for epidemiological studies of candidiasis in immunocompromised hosts. Journal of Infectious Diseases 159, 488-494.

Fukuda, Y., Atomi, H., Kurihara, T., Hikida, M., Ueda, M. \& TANAKA, A. (1991). Genes encoding peroxisomal enzymes are not necessarily assigned on the same chromosome of an n-alkaneutilizable yeast Candida tropicalis. FEBS Letters 286, 61-63.

Iwaguchi, S., Homma, M. \& TanakA, K. (1990). Variation in electrophoretic karyotype analysed by assignment of DNA probes in Candida albicans. Journal of General Microbiology 136, 24332442.

Kamiyama, A., Nimi, M., Tokunaga, M. \& Nakayama, H. (1989). Adansonian study of Candida albicans: intraspecific homogeneity excepting $C$. stellatoidea strains. Journal of Medical and Veterinary Mycology 27, 229-241.

KoHne, D. E., Chiscon, T. A. \& Hoyer, B. H. (1972). Evolution of primate DNA sequences. Journal of Human Evolution 1, 627644.

Kurtzman, C. P., Smiley, M. J. \& Johnson, C. J. (1980). Emendation of the genus Issatchenkia Kudriavzev and comparison of species by deoxyribonucleic acid reassociation, mating reaction, and ascospore ultrastructure. International Journal of Systematic Bacteriology 30, 503-513.

Kwon-Chung, K. J., Riggsby, W. S., Uphoff, R. A., Hicks, J. B., Whelan, W. L., Reiss, E., Magee, B. B. \& Wickes, B. L. (1989). 
Genetic differences between Type I and Type II Candida stellatoidea. Infection and Immunity 57, 527-532.

Kwon-Chung, K. J., Wickes, B. L. \& Merz, W. G. (1988). Association of electrophoretic karyotype of Candida stellatoidea with virulence for mice. Infection and Immunity 56, 1814-1819.

Kwon-ChUNG, K. J., HICKs, J. B. \& LIPKE, P. N. (1980). Evidence that Candida stellatoidea Type II is a mutant of Candida albicans that does not express sucrose-inhibitable alpha-glucosidase. Infection and Immunity 9, 2804-2808.

LODDER, J. \& KREGER-VAN RiJ, N. J. W. (1952). Candida Berkhout. In The Yeasts, a Taxonomic Study, pp. 578-581. Amsterdam: NorthHolland Publishing Co.

Maniatis, T., Fritsch, E. F. \& SAmbrook, J. (1982). Molecular Cloning: a Laboratory Manual. Cold Spring Harbor, NY: Cold Spring Harbor Laboratory.

Martin, D. S., Jones, C. P., Yao, K. F. \& Lee, L. E., JR (1937). A practical classification of the Monilias. Journal of Bacteriology 35, 99-123.

MEYer, S. A. (1979). DNA relatedness between physiologically similar strains and species of yeasts of medical and industrial importance. In Single-cell Protein: Safety for Animal and Human Feeding, pp. 13-19. Edited by S. Garatini, S. Paglialunga \& N. S. Serimshaw. New York: Pergamon.

ODDs, F. C. (1987). Candida infections: an overview. CRC Critical Reviews in Microbiology 156, 471-477.

PiCataggio, S., Deanda, K. \& Mielenz, J. (1991). Determination of Candida tropicalis acyl coenzyme A oxidase isozyme function by sequential gene disruption. Molecular and Cellular Biology 11, 43334339.
SCherer, S. \& Stevens, D. A. (1988). A Candida albicans dispersed, repeated gene family and its epidemiologic applications. Proceedings of the National Academy of Sciences of the United States of America 85, 1452-1456.

Soll, D. R., Staebell, M., langtim, C., Pfaller, M., Hicks, J. \& Gopala RaO, T. V. (1988). Multiple Candida strains in the course of a single systemic infection. Journal of Clinical Microbiology 26, 1448-1459.

Suzuki, T., Kobayashi, I., Mizuguchi, I., Banno, I. \& Tanaka, K. (1988). Electrophoretic karyotypes in medically important Candida species. Journal of General and Applied Microbiology 34, 409-416.

SuZUKI, T., MiYamae, Y. \& IshidA, I. (1991). Variation in colony morphology and chromosomal rearrangement in Candida tropicalis. Journal of General Microbiology 137, 161-167.

van UdEn, N. \& BuCKLey, H. (1970). Candida Berkhout. In The Yeasts, a Taxonomic Study, pp. 989-991. Edited by J. Lodder. Amsterdam: North-Holland Publishing Co.

VARMA, A. K. \& KWON-ChUNG, K. J. (1991). A rapid method to extract DNA from Cryptococcus neoformans. Journal of Clinical Microbiology 29, 810-812.

Wickes, B. L., Golin, J. E. \& Kwon-Chung, K. J. (1991a). Chromosomal rearrangement in Candida stellatoidea results in a positive effect on phenotype. Infection and Immunity 59, 17621771.

Wickes, B. L., Staudinger, J., Magee, B. B., Kwon-Chung, K. J., MAGEe, P. T. \& SCHERER, S. (1991b). Physical and genetic mapping of Candida albicans: several genes previously assigned to chromosome 1 map to chromosome $\mathrm{R}$, the rDNA-containing linkage group. Infection and Immunity 59, 2408-2484. 